\title{
CENTRALISER NEAR-RING REPRESENTATIONS
}

\author{
by C. J. MAXSON and K. C. SMITH \\ (Received 7th August 1980)
}

\section{Introduction}

Let $V$ be a group, written additively but not necessarily abelian, and let $S$ be a semigroup of endomorphisms of $V$. The set $C(S ; V)=\{f: V \rightarrow V \mid f \sigma=\sigma f$ for all $\sigma \in S$ and $f(0)=0\}$ forms a zero-symmetric near-ring with identity under the operations of function addition and composition, called the centraliser near-ring determined by $S$ and $V$. Centraliser near-rings are very general, for if $N$ is any zero-symmetric near-ring with 1 then there exists a group $V$ and a semigroup $S$ of endomorphisms of $V$ such that $N \simeq C(S ; V)$.

In this paper all near-rings will be finite, zero-symmetric and have an identity element. For definitions and results concerning near-rings see Pilz [11].

The first centraliser near-ring representation result was given by Wielandt [13]. Here he announced the characterisation of finite simple near-rings as centraliser near-rings $C(\mathscr{A} ; V)$ where $\mathscr{A}$ is a group of fixed point free automorphisms of the group $V$. In 1973 , Betsch [2] extended Wielandt's work to a class of infinite near-rings. Recently, there have been several investigations into the structure of centraliser near-rings. (See [6], [7], [8] and [9].) In [7] we established the following result.

Theorem 1.1. Let $V$ be a finite group and $\mathscr{A}$ a group of automorphisms of $V$. Then $C(\mathscr{A} ; V)$ is simple if and only if the stabiliser subgroups, $\operatorname{stab}_{\mathscr{A}}(v) \equiv\{\alpha \in \mathscr{A} \mid \alpha v=v\}$, are conjugate for all $v \in V^{*} \equiv V-\{0\}$.

Our investigations in this paper are concerned with the following representation question. If $N$ is a simple subnear-ring of $C(\{1\}) ; V)$, when is $N=C(\mathscr{A} ; V)$ for some $\mathscr{A} \subseteq$ Aut $V$ ? Equivalently, let $V$ be a near-ring module over the simple near-ring $N$ and for $a \in N$ define $\lambda_{a}: V \rightarrow V$ by $\lambda_{a} v=a v, v \in V$. Then $N$ is isomorphic to $\bar{N} \equiv\left\{\lambda_{a} \mid a \in N\right\} \subseteq C(\{1\} ; V)$, and we interpret a representation of $N$ as a $C(\mathscr{A} ; V)$ to mean a representation of $\bar{N}$.

This centraliser representation problem is the non-linear analogue of the following ring theory problem. Let $V$ be an abelian group and let $S$ be a simple subring of End $V$. When does there exist a ring $R$ such that $S=$ End $_{R} V$ ? A partial solution to this problem is a consequence of the Noether-Skolem Theorem [4], page 104, in the setting where End $V$ is simple.

We now give a short summary of our results. In the next section we consider the general representation problem giving necessary and sufficient conditions for a centraliser near-ring representation of a simple near-ring $N$. In Section 3 we apply these 
results to near-fields and fields where the representation problem is discussed under various situations.

\section{Characterisation Theorems}

In this section we give necessary and sufficient conditions on a simple near-ring $N$, $N \subseteq C(\{1\} ; V)$, in order that $N=C(\mathscr{A} ; V)$ for some $\mathscr{A} \subseteq$ Aut $V$.

Lemma 2.1. Let $N$ be a simple subnear-ring of $C(\{1\} ; V)$ and let $B=\mathrm{Aut}_{N} V$. If there exists $v_{1}, \ldots, v_{t} \in V$ such that $\left\{v_{1}, \ldots, v_{t}\right\} \subseteq \theta_{B}\left(v_{1}\right)$ where $\theta_{B}\left(v_{1}\right)$ is the orbit of $V$ containing $v_{1}$ determined by the action of $B$ on $V$, and $V=N v_{1} \dot{\cup} \ldots \dot{\cup} N v_{t}$ (disjoint union) where each $N v_{i}$ is a faithful $N$-simple, $N$-subgroup of $V$, then $C(B ; V)$ is simple.

Proof. We show first that elements of $N v_{i}$ have the same $B$-stabiliser. Let $v$ be a nonzero element in $N v_{i}$. Since $N v_{i}$ is $N$-simple, $N v=N v_{i}$. If $\alpha \in \operatorname{stab}_{B}(v)$ then $\alpha(v)=v$ and $\alpha\left(N v_{i}\right)=N v_{i}$. Thus $\alpha$ restricted to $N v_{i}$ is an $N$-automorphism of $N v_{i}$ fixing $v$. Since $N v_{i}$ is $N$-simple $\alpha$ must be the identity map on $N v_{i}$. From this, we conclude that $\operatorname{stab}_{B} v$ $=\operatorname{stab}_{B} v_{i}$ for all $v \in N v_{i}$.

Let $\theta_{B}(w)$ be any $B$-orbit. Since $w \in N v_{j}$ for some $j$, then $w=n v_{j}$ for some $n \in N$. But each $v_{i}$ belongs to the same $B$-orbit so $\alpha v_{j}=v_{i}$ for some $\alpha \in B$. Hence $\alpha w=\alpha n v_{j}=n \alpha v_{j}$ $=n v_{i}$. This means $\theta_{B}(w) \cap N v_{i} \neq \emptyset$ for each $i$. Since all $B$-stabilisers of elements in $\theta_{B}(w)$ are conjugate and since $\theta_{B}(w)$ intersects every $N v_{i}$ then any two $B$-stabilisers are conjugate which implies that $C(B ; V)$ is simple.

This leads to the main characterisation result.

Theorem 2.1. Let $N$ be a simple subnear-ring of $C(\{1\} ; V)$, and let $B=A^{2}{ }_{N} V$. Then the following are equivalent.

(1) $N=C(\mathscr{A} ; V)$ for some $\mathscr{A} \subseteq$ Aut $V$.

(2) $N=C(B ; V)$.

(3) i. $V=N v_{1} \dot{\cup} \ldots \dot{\cup} N v_{t}$ where each $N v_{i}$ is a faithful $N$-simple $N$-subgroup of $V$ and each $v_{i} \in \theta_{B}\left(v_{1}\right)$.

ii. Let $S_{1}=\operatorname{stab}_{B}\left(v_{1}\right)$, then Fix $S_{1} \equiv\left\{v \in V \mid \alpha v=v\right.$ for all $\left.\alpha \in S_{1}\right\}$ is a subset of $N v_{1}$.

Part 3ii may be replaced by 3ii': Fix $S_{1}$ is $N$-simple.

Proof. If part (1) is true then $\mathscr{A} \subseteq B$ and so $C(B ; V) \subseteq C(\mathscr{A} ; V)$. But by definition of $B$, $N \subseteq C(B ; V)$ and hence (1) implies (2).

If (2) is true then $N=C(B ; V)$ and $N$ is simple. Select a nonzero $v \in V$ then $N v$ $=\left\{w \in V \mid \operatorname{stab}_{B} w=\operatorname{stab}_{B} v\right\} \cup\{0\}$. Because $N=C(B ; V)$ then $N$ acts transitively on the nonzero elements of $N v$ (see [7]). Hence $N v$ is $N$-simple. Moreover there exist elements $v_{1}, \ldots, v_{t}$ all in $\theta_{B}\left(v_{1}\right)$ such that $V=N v_{1} \dot{U} \ldots \dot{\cup} N v_{t}$ and $N v_{1}=$ Fix $S_{1}$ (see [7]).

Assume (3) is true. Then Lemma 2.1 implies $C(B ; V)$ is simple. Hence Fix $S_{1}$ $=C(B ; V) v_{1}$. We have $N v_{1} \subseteq C(B ; V) v_{1}=$ Fix $S_{1}$, and by (3)ii (or (3)ii'), $N v_{1}=C(B ; V) v_{1}$. But $C(B ; V) v_{1}$ is the set of all possible images of $v_{1}$ under functions in $C(B ; V)$ and $N v_{1}$ 
is the set of all possible images of $v_{1}$ under functions from $N$, and by assumption $v_{1}, \ldots, v_{t}$ belong to the same $B$-orbit. So $N=C(B ; V)$ as desired. Since (2) implies (1) is obvious, the proof is complete.

In the following theorem we establish the existence of near-rings $C(\{1\} ; V)$ which contain simple subnear-rings that are not centraliser representable on $V$.

Theorem 2.2. Let $N$ be a simple near-ring. Then there exists an $N$-module $V$ such that $N$ has no representation as a centraliser near-ring on $V$.

Proof. It is shown in [5] that if $V$ is a group and $\mathscr{A}$ a group of automorphisms of $V$ then $C(\mathscr{A} ; V)$ is a simple ring if and only if $C(\mathscr{A} ; V)$ is a field. Hence a simple ring which is not a field has no centraliser representation. If $N$ is a field then Theorem 3.2 at the end of this paper applies. So we may assume $N$ is a simple nonring.

From [2] we have the representation $N=C(\mathscr{A} ; W)$ where $\mathscr{A}$ is a group of automorphisms acting fixed point free on $W$. Let $V=W+W$, and for each $f \in N$ extend $f$ to all of $V$ by defining $f\left(\begin{array}{l}x \\ y\end{array}\right)=\left(\begin{array}{l}f(x) \\ f(y)\end{array}\right)$. In this way $V$ is an $N$-module and we may regard $N$ as a subnear-ring of $C(\{1\} ; V)$. We will show that $N$ has no centraliser representation on $V$.

Assume first that $N$ is not a near-field. Then under the action of $\mathscr{A}, W$ has at least two nonzero orbits. Let $w_{1}, w_{2} \in W$ be nonzero elements belonging to different orbits. We have, as sets, $N\left(\begin{array}{c}w_{1} \\ 0\end{array}\right)=\left(\begin{array}{c}N w_{1} \\ 0\end{array}\right)$ and $N\left(\begin{array}{c}w_{1} \\ w_{2}\end{array}\right)=N\left(\begin{array}{c}w_{1} \\ 0\end{array}\right)+N\left(\begin{array}{c}0 \\ w_{2}\end{array}\right)=\left(\begin{array}{c}N w_{1} \\ 0\end{array}\right)+\left(\begin{array}{c}0 \\ N w_{2}\end{array}\right)=\left(\begin{array}{c}N w_{1} \\ N w_{2}\end{array}\right)$. The cardinality $\left|\left(\begin{array}{c}N w_{1} \\ N w_{2}\end{array}\right)\right|$ of $N\left(\begin{array}{c}w_{1} \\ w_{2}\end{array}\right)$ is greater than the cardinality $\left|\left(\begin{array}{c}N_{w_{1}} \\ 0\end{array}\right)\right|$ of $N\left(\begin{array}{c}w_{1} \\ 0\end{array}\right)$, so $N$ cannot be centraliser representable on $V$ since part (3)i of Theorem 2.1 implies that $|N v|=|N w|$ for nonzero $v, w \in V$.

Now assume $N$ is a near-field. Then $W=(N,+)$ and $\mathscr{A}$ consists of the right multiplication maps by elements of $N$ on $W$. The linear maps in $N$ acting on $V=W \dot{+} W$ are $N_{L}=\left\{\left(\begin{array}{l}f \\ 0\end{array}\right) \mid f \in N\right.$ is linear on $\left.W\right\}$. A calculation shows that $\operatorname{End}_{N_{L}} V$ $\left.=\left\{\left(\begin{array}{c}\alpha_{1} \alpha_{2} \\ \alpha_{3} \alpha_{4}\end{array}\right) \mid \alpha_{i} \in \mathscr{A} \cup: 0\right\}\right\}$. We show now that Aut $_{N} V=\left\{\left(\begin{array}{cc}\alpha & 0 \\ 0 & \beta\end{array}\right) \mid \alpha, \beta \in \mathscr{A}\right\} \cup\left\{\left(\begin{array}{cc}0 & \alpha \\ \beta & 0\end{array}\right) \mid \alpha, \beta \in \mathscr{A}\right\}$. For suppose $B=\left(\begin{array}{c}\alpha_{1} \alpha_{2} \\ a_{3} \alpha_{4}\end{array}\right) \in \mathrm{Aut}_{N} V$ with say $\alpha_{1} \neq 0, \alpha_{2} \neq 0$. Then for every $f \in N,\left(\begin{array}{l}x \\ y\end{array}\right) \in V$,

or

$$
f B\left(\begin{array}{l}
x \\
y
\end{array}\right)=B f\left(\begin{array}{l}
x \\
y
\end{array}\right)
$$

$$
\begin{aligned}
f\left(\alpha_{1} x+\alpha_{2} y\right) & =\alpha_{1} f(x)+\alpha_{2} f(y) \\
& =f\left(\alpha_{1} x+\alpha_{2} y\right)
\end{aligned}
$$

for every $x, y \in W$. But since $\alpha_{1}, \alpha_{2}$ are invertible this implies $f$ acts linearly on $W$ and $C(\mathscr{A} ; W)$ is a field. Hence one of $\alpha_{1}, \alpha_{2}$ must be 0 . Similarly for $\alpha_{3}, \alpha_{4}$ and $B$ has the desired form.

It remains to show that $N \neq C\left(\right.$ Aut $\left._{N} V ; V\right)$. This is done by showing the latter is not simple. Let $\overline{\mathscr{A}}=$ Aut $_{N} V$ and let $w$ be a nonzero element of $W$. Then $\operatorname{stab}_{\bar{d}}\left(\begin{array}{c}w \\ 0\end{array}\right)$

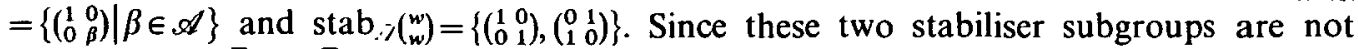
conjugates in $\overline{\mathscr{A}}, C(\overline{\mathscr{A}} ; V)$ is not simple.

Turning now to near-fields $N$ in $C(\{1\} ; V)$, the characterisations as to when $N$ is a centraliser near-ring on $V$ appear tighter. We again fix some notation. As above let $B$ 
$=$ Aut $_{N} V$ and let $S_{0}=\operatorname{stab}_{B}\left(v_{0}\right)$ for fixed nonzero $v_{0}$ in $V$. Also let Fix $S_{0}=\{v \in V \mid \alpha v=v$ for each $\left.\alpha \in S_{0}\right\}$ and $\mathscr{N}_{0}=$ normaliser of $S_{0}$ in $B$.

Corollary 2.1. Let $N$ be a near-field in $C(\{1\}, V)$. The following are equivalent.

(1) $N=C(\mathscr{A} ; V)$ for some $\mathscr{A} \subseteq$ Aut $V$.

(2) $N=C(B ; V)$.

(3) $B$ is transitive on $V$ and Fix $S_{0} \subseteq N v_{0}$.

(4) $B$ is transitive on $V$ and Fix $S_{0}$ is $\mathrm{N}$-simple.

(5) $B$ is transitive on $V$ and $\mathscr{N}_{0} / S_{0}$ is isomorphic to $N^{*}$.

Proof. That (1) is equivalent to (2) is clear. Assume $N=C(B ; V)$. Since $N$ is a nearfield, $B$ is transitive on $V$ (see [7]), and Fix $S_{0} \subseteq N v_{0}$ from Theorem 2.1. So (2) implies (3).

To show (3) implies (4), we note that the transitivity of $B$ implies that $C(B ; V)$ is a near-field and so Fix $S_{0}=C(B ; V) v_{0}$. But then Fix $S_{0}=N v_{0}$. If $H$ is a nonzero $N$ subgroup of Fix $S_{0}$ then for some $n \in N, n v_{0}=h \in H$. But then $v_{0}=n^{-1} h$ is in $H$ and so $H$ $=N v_{0}=$ Fix $S_{0}$. Hence Fix $S_{0}$ is $N$-simple.

Assume (4) is true. Then by Theorem $2.1 N=C(B ; V)$, and from [7] Theorem 3, $N \cong C\left(\mathscr{N}_{0} / S_{0} ;\right.$ Fix $\left.S_{0}\right)$ where $\mathscr{N}_{0} / S_{0}$ acts fixed point free on Fix $S_{0}$. But also $N \cong C\left(N^{*} ; N\right)$ and by the isomorphism result of Ramakotaiah $[12], \mathscr{N}_{0} / S_{0} \cong N^{*}$. So (4) implies (5).

If (5) holds then $N \subseteq C(B ; V)$ with $C(B ; V)$ a near-field. If $K=C(B ; V)$ then $K^{*} \cong \mathscr{N}_{0} / S_{0}$ and thus $\left|N^{*}\right| \leqq\left|K^{*}\right| \leqq\left|\mathcal{N}_{0} / S_{0}\right|=\left|N^{*}\right|$. So $N=K$ as desired.

If $N \subseteq C(\{1\} ; V)$ is a near-field and if $\mathrm{Aut}_{N} V$ is transitive on $V$ then $C\left(\operatorname{Aut}_{N} V ; V\right)$ is a near-field containing $N$. The following example shows that $N$ need not equal $C\left(\mathrm{Aut}_{\boldsymbol{N}} \boldsymbol{V} ; \mathrm{V}\right)$.

Example 2.2. Let $N=D(5,2,3)$ be a Dickson near-field of order $5^{6}$ with centre of order $5^{2}$. Then the field $N_{1}=D(5,3,1)$ is a subnear-field of $N$ of order $5^{3}$ (see DancsGrove [3]). Let $V=\langle N,+\rangle$, the additive group of $N$ (or of the Galois field $G F\left(5^{6}\right)$ ). The field $N_{1}$ acts on $V$ by left multiplication so we may regard $N_{1}$ (and $N$ ) as subnear-rings of $C(\{1\}, V)$. Since $\operatorname{Aut}_{N_{1}} V$ contains $\left\{\rho_{n}: V \rightarrow V \mid \rho_{n}(v)=v n, n \in N, v \in V\right\}$, Aut $_{N_{1}} V$ is transitive on $V$. We have $N_{1} \subseteq C\left(\right.$ Aut $\left._{N_{1}} V ; V\right) \subseteq C\left(\right.$ Aut $\left._{N} V ; V\right)=N$ and since $N_{1}$ is a maximal subnear-field of $N$ (Dancs-Grove [3]) then either $N_{1}=C\left(\right.$ Aut $\left._{N_{1}} V ; V\right)$ or $N$ $=C\left(\right.$ Aut $\left._{N_{1}} V ; V\right)$. We will show that the latter is true.

If $N_{1}=C\left(\right.$ Aut $\left._{N_{1}} V ; V\right)$ then Aut $_{N_{1}} V$ is not fixed point free on $V$ since $\left|\mathrm{Aut}_{N_{1}} V\right|>\left|N_{1}\right|$. Thus there exists a $\Phi \in$ Aut $_{N_{1}} V$ such that $\Phi \neq 1$ and $\Phi(1)=1$. We will show this is impossible.

Using the notation of Pilz [11], page 244, let $g$ be a generator of the multiplicative cyclic group $G F\left(5^{6}\right)^{*}$ used in the construction of $N$. Let $H$ be the subgroup of $G F\left(5^{6}\right)^{*}$ of index 3 generated by $g^{3}$ and let $\sigma$ be the Galois automorphism of $G F\left(5^{6}\right)$ defined by $x \rightarrow x^{5^{2}}$. The cosets of $H$ in $G F\left(5^{6}\right)^{*}$ are $H, H g, H g^{2}$ and the multiplication in $N$ is defined in terms of the multiplication in $G F\left(5^{6}\right)$ by $a \circ b=a^{\sigma^{i}} b$ if $b \in H g^{i}$ and $a \circ 0=0$. 
If $\Phi \in$ Aut $_{N_{1}} V$ is as described above we will show that $\Phi$ is $G F\left(5^{-3}\right)$-linear on $V$ as a vector space over $G F\left(5^{3}\right)$ and that $\Phi\left(H g^{i}\right)=H g^{i}, i=0,1,2$.

Since $V$ has dimension 2 over $G F\left(5^{3}\right)$ and since $g^{3} \notin G F\left(5^{3}\right)$ then $\left\{1, g^{3}\right\}$ forms a basis for $V$. So every element in $V$ has the form $\alpha 1+\beta g^{3}, \alpha, \beta \in G F\left(5^{3}\right)$. But $\alpha 1+\beta g^{3}=\alpha \circ 1$ $+\beta \circ g^{3}, \alpha, \beta \in N_{1}$ since $\left\{1, g^{3}\right\} \subseteq H$. If $\delta \in N_{1}$ then

$$
\left.\delta \circ \Phi\left(\alpha \circ 1+\beta \circ g^{3}\right)=\delta \circ\left(\alpha+\beta \circ \Phi\left(g^{3}\right)\right)=\delta^{\sigma^{i}}\left(\alpha+\beta \circ \Phi\left(g^{3}\right)\right)=\delta^{\sigma^{i}} \alpha+\delta^{\sigma^{i}}\left(\beta \circ \Phi g^{3}\right)\right)
$$

where $\alpha+\beta \circ \Phi\left(g^{3}\right) \in H g^{i}$. On the other hand

$$
\begin{aligned}
\delta \circ \Phi\left(\alpha \circ 1+\beta \circ g^{3}\right) & =\Phi\left(\delta \circ\left(\alpha \circ 1+\beta \circ g^{3}\right)\right)=\Phi\left(\delta^{\sigma^{j}}\left(\alpha \circ 1+\beta \circ g^{3}\right)\right) \\
& =\Phi\left(\delta^{\sigma^{j}} \alpha 1+\delta^{\sigma^{j}}\left(\beta \circ g^{3}\right)\right)=\delta^{\sigma^{j}} \alpha+\Phi\left(\delta^{\sigma^{j}} \beta g^{3}\right) \\
& =\delta^{\sigma^{j}} \alpha+\Phi\left(\left(\delta^{\sigma^{j}} \beta\right) \circ g^{3}\right)=\delta^{\sigma^{j}} \alpha+\delta^{\sigma^{j}} \beta \circ \Phi\left(g^{3}\right)
\end{aligned}
$$

where $\alpha \circ 1+\beta \circ g^{3} \in H g^{j}$. Comparing the two results and using the fact that $\Phi\left(g^{3}\right) \notin N_{1}$ we conclude that if $\alpha \neq 0$ then $\delta^{\sigma^{i}}=\delta^{\sigma^{j}}$, and so $\sigma^{i}=\sigma^{j}$. This means $\Phi$ preserves $H g, H g^{2}$ and thus $H$. If $a \in N_{1}, v \in V$ then since $\Phi$ preserves cosets $\Phi(a \circ v)=\Phi\left(a^{\sigma^{i}} v\right)$ while $a \circ \Phi(v)$ $=a^{\sigma^{i}} \Phi(v)$. So $a^{\sigma^{i}} \Phi(v)=\Phi\left(a^{\sigma^{i}} v\right)$ for all $a \in G F\left(5^{3}\right)$. Hence $\Phi$ is $G F\left(5^{3}\right)$-linear on $V$.

To finish the example it suffices to show that the two dimensional vector space $V$ (the additive group of $G F\left(F^{6}\right)$ ) over $G F\left(5^{3}\right)$ has no nontrivial one-to-one $G F\left(5^{3}\right)$-linear maps $\Phi$ which preserve the cosets of $H$ in $G F\left(5^{3}\right)^{*}$ and fix 1 . This is done in the following lemma due to Martin R. Pettet.

Lemma 2.2. (M. R. Pettet) Let $H$ be the subgroup of $G F\left(5^{6}\right)^{*}$ of index 3. If $\Phi: G F\left(5^{6}\right) \rightarrow G F\left(5^{6}\right)$ is a $G F\left(5^{3}\right)$-linear group automorphism which preserves the cosets of $H$ in $G F\left(5^{6}\right)^{*}$ and such that $\Phi(1)=1$, then $\Phi=1$.

Proof. Assume such a $\Phi$ exists with $\Phi \neq 1$. Then there exists such a $\Phi$ whose order is a prime $p$, i.e. $\Phi^{p}=1$. Since $G F\left(5^{3}\right)^{*} \subseteq H, H \cup\{0\}$ is the union of 42 one dimensional $G F\left(5^{3}\right)$-subspaces, one of which is $G F\left(5^{3}\right)$. Since $\Phi$ leaves $G F\left(5^{3}\right)$ fixed it permutes the other 41 subspaces in $H$. If $\Phi$ does not leave another subspace fixed then, since 41 is a prime, $\Phi$ permutes the 41 subspaces cyclically which means that $p=41$. But $p=41$ is impossible since 41 does not divide the order of $G L\left(V / G F\left(5^{3}\right)\right)$, i.e. there is no $G F\left(5^{3}\right)$ automorphism of $V$ of order 41 . So $\Phi$ leaves $G F\left(5^{3}\right)$ and at least one other subspace in $H \cup\{0\}$ invariant. Hence $\Phi$ has two linearly independent eigenvectors, say $\{1, \alpha\}$ and the matrix of $\Phi$ with respect to this basis for $V$ is $\left(\begin{array}{l}1 \\ 0 \\ c\end{array}\right), c \in G F\left(5^{3}\right)^{*}$. Since $\Phi^{p}=1$ then $c^{p}=1$ and so $p$ divides $\left|G F\left(5^{3}\right)^{*}\right|=2^{2} .31$, hence $p=2$ or $p=31$. If $p=31$ then there would be 42 $-31=11$ fixed subspaces in $H \cup\{0\}$ resulting in too many distinct eigenvectors. Thus $p=2$ and the matrix of $\Phi$ is $\left(\begin{array}{cc}1 & 0 \\ 0 & -1\end{array}\right)$.

Since $\Phi$ preserves the cosets of $H$ this means that $a+b \alpha \equiv a-b \alpha \bmod H$ for every $a, b \in G F\left(5^{3}\right)$, not both zero. The above is clearly true if $b=0$ so if $b \neq 0$ we have $(a+\alpha)(a$ $-\alpha)^{-1} \in H$ for every $a \in G F\left(5^{3}\right)$. The map $a \rightarrow(a+\alpha)(a-\alpha)^{-1}$ from $G F\left(5^{3}\right)$ into $H$ is oneto-one. Since there are 125 such elements $(a+\alpha)(a-\alpha)^{-1}$ in $H$ and $42 G F\left(5^{3}\right)$-subspaces 
in $H \cup\{0\}$ there is at least one subspace containing three elements of the form $(a+\alpha)(a$ $-\alpha)^{-1}$, say $(a+\alpha)(a-\alpha)^{-1},(b+\alpha)(b-\alpha)^{-1},(c+\alpha)(c-\alpha)^{-1}$ where

$$
(a+\alpha)(a-\alpha)^{-1}=f_{1}(b+\alpha)(b-\alpha)^{-1}=f_{2}(c+\alpha)(c-\alpha)^{-1},
$$

$a, b, c, f_{1}, f_{2} \in G F\left(5^{3}\right)$, all distinct and $f_{1} \neq 1 \neq f_{2}$. From the above we have

and

$$
\left(f_{1}-1\right) \alpha^{2}=\left(f_{1}+1\right)(a-b) \alpha+\left(f_{1}-1\right) a b
$$

$$
\left(f_{2}-1\right) \alpha^{2}=\left(f_{2}+1\right)(a-c) \alpha+\left(f_{2}-1\right) a c .
$$

This gives two quadratic polynomials over $G F\left(5^{3}\right)$ having $\alpha$ as a root. Since $\alpha \notin G F\left(5^{3}\right)$ these two polynomials give rise to the same minimal polynomial. From this we have $a b$ $=a c$, so $b=c$ or $a=0$. The latter is impossible since the polynomials are irreducible. But $b=c$ is also impossible since $(b+\alpha)(b-\alpha)^{-1} \neq(c+\alpha)(c-\alpha)^{-1}$. This contradiction shows $\Phi=1$ as desired.

\section{Specialised actions and further examples}

We. now apply the characterisation theorem of the previous section to obtain results on specified actions of a near-ring $N$ on an $N$-module $V$. Recall that when $N$ is a nearfield, $V$ is a near-vector space over $N$ if $V=V_{1} \oplus \cdots \oplus V_{t}$ where each $V_{i}$ is an $N$ submodule of $V$ and $V_{i} \cong N$ (see [1]).

Theorem 3.1. Let $N$ be a near-field and $V$ a near-vector space over $N$. Then $N$ $=C(\mathscr{A} ; V)$ for some $\mathscr{A} \subseteq$ Aut $V$.

Proof. From the results of Beidleman [1] it is easy to see that Aut ${ }_{N} V$ is transitive on $V$. Also if $v_{1} \in V_{1}$, then for $S_{1}=\operatorname{stab}\left(v_{1}\right)$ we have Fix $S_{1}=V_{1}=N v_{1}$. Thus by Theorem $2.1, N=C(\mathscr{A} ; V)$ as desired.

Corollary 3.1. Let $F$ be a field, $F \subseteq C(\{1\}, V)$. If $F$ acts linearly on $V$ then $F=C(\mathscr{A} ; V)$ for some $\mathscr{A} \subseteq$ Aut $V$.

Proof. If $F$ acts linearly on $V$ then $V$ is a vector space over $F$ and the theorem applies.

Corollary 3.2. Let $F$ be a field and let $V$ be a monogenic near-ring module over $F$. Then $F=C(\mathscr{A} ; V)$ for some $\mathscr{A} \subseteq$ Aut $V$.

Proof. Let $V=F v_{0}$. Then for $h \in F, w, u \in V$ we have $w=f v_{0}, u=g v_{0}$ for some $f, g \in F$ and $h(w+u)=h\left(f v_{0}+g v_{0}\right)=h(f+g) v_{0}=(h f+h g) v_{0}=h w+h u$. Thus $F$ acts linearly on $V$ and Corollary 3.1 applies.

Còrollary 3.3. Let $F$ be a field acting on $V$. Then $F \subseteq C(B ; V)$ where $B=\mathrm{Aut}_{F} V$. If $B$ is a p-group acting transitively on $V$ then $F=C(B ; V)$. 
Proof. From Passman [10], page 34, either $B$ is a cyclic group or else $|V|=3^{2}$. If $B$ is cyclic, then from Maxson and Smith [6],B acts fixed point free on $V$ and $C(B ; V)$ is a field. Since $V$ is $C(B ; V)$-monogenic, $C(B ; V)$ acts linearly on $V$ and thus so does $F$. Hence $F=C(B ; V)$.

Suppose $|V|=3^{2}$, and let $v_{0} \in V^{*}$. If $S=\operatorname{stab} v_{0}$, we have Fix $S=C(B ; V) v_{0}$ and since $\mid$ Fix $S \mid$ divides $\left|V^{*}\right|=3^{2}-1$ then $\mid$ Fix $S \mid=3^{l}-1$ where $l=1$ or 2 . If $l=1$ then $|C(B ; V)|=3$ and $C(B ; V)=G F(3)$ acts linearly on $V$. If $l=2, V$ is $C(B ; V)$-monogenic and again $F$ acts linearly on $V$ so $F=C(B ; V)$ as desired.

From the above corollaries it is natural to conjecture that for a field $F$ acting on $V, F$ $=C(\mathscr{A} ; V)$ implies $V$ is a vector space over $F$. This is false, however, as the next example, due to $\mathrm{S}$. Gagola, provides a field acting non-linearly on $V$ but $F=C(\mathscr{A} ; V)$.

Example 3.1. Let $V=G F\left(p^{4}\right)$, where $p$ is a prime different from 3 and let $\sigma$ be the Galois automorphism $x \rightarrow x^{p}$ of $G F\left(p^{4}\right)$. For $a \in G F\left(p^{4}\right)^{*}$ and $i=0,1,2,3$ define the maps $T_{a, \sigma^{i}}: V \rightarrow V$ by $T_{a, \sigma^{i}} v=a v^{\sigma^{i}}$. It is easy to verify that $\mathscr{F}=\left\{T_{a, \sigma^{i}} \mid a \in G F\left(p^{4}\right)^{*}, i=0,1,2,3\right\}$ is a group of automorphisms of $V$. Let

$$
\mathscr{A}=\left\{T_{a, \sigma^{i}} \mid \text { if } a \text { is a square in } G F\left(p^{4}\right)^{*}\right.
$$

then $i=0,2$ while if $a$ is not a square then $i=1,3\}$,

a subgroup of $\mathscr{F}$. Since $\mathscr{A}$ is a transitive automorphism group then $C(\mathscr{A} ; V)$ is a nearfield. Also $S \equiv \operatorname{stab}(1)=\left\{T_{1, \sigma^{i}} \mid i=0,2\right\}$ and Fix $S=G F\left(p^{2}\right) \subset V=G F\left(p^{4}\right)$. If $N(S)$ is the normaliser of $S$ in $\mathscr{A}$ then it is easy to verify that $N(S) / S \cong G F\left(p^{2}\right)^{*}$. Thus $C(\mathscr{A} ; V) \cong G F\left(p^{2}\right)$.

We now show that the field $C(\mathscr{A} ; V)$ does not act linearly on $V$. Suppose $f \in C(\mathscr{A} ; V)$. Then $f T_{a, \sigma^{i}}=T_{a, \sigma^{i}} f$ implies $f(a)=a f(1)^{\sigma^{i}}$. If $a$ is a square then $f(a)=a f(1)$, while if $a$ is not a square then $f(a)=a f(1)^{\sigma}$. Thus $f$ is completely determined by its action on 1 . Since $1 \in$ Fix $S$ we have $f(1) \in$ Fix $S$. Now suppose $f \in C(\mathscr{A} ; V)$ acts linearly on $V$. Suppose $b \in V$ is not a square. Consider $1+b$. If $1+b$ is a square then $f(1+b)=(1$ $+b) f(1)=f(1)+b f(1)$, while $f(1)+f(b)=f(1)+b f(1)^{\sigma}$. Comparing the two results gives $f(1)^{\sigma}=f(1)$, or $f(1) \in G F(p)$. If $1+b$ is not a square then $f(1+b)=(1+b) f(1)^{\sigma}=f(1)^{\sigma}$ $+b f(1)^{\sigma}$, while $f(1)+f(b)=f(1)+b f(1)^{\sigma}$. Again $f(1) \in G F(p)$. Hence $f \in C(\mathscr{A} ; V)$ is linear on $V$ if and only if $f(1) \in G F(p)$. Therefore the field $C(\mathscr{A} ; V)$ does not act linearly on $V$.

We conclude by defining a class of actions of a field $F$ on a vector space $V$ that cannot give rise to centraliser near-rings. But first a lemma from linear algebra.

Lemma 3.1. (S. Gagola) Let $V$ be a finite dimensional vector space over a finite field $F$, and let $W, Y$ be proper subspaces of $V$. If $F=G F(2)$, assume one of $W$ and $Y$ is not a maximal subspace. Then there is a basis $B$ of $V$ such that $B \subseteq V-(W \cup Y)$.

Proof. If $F=G F(2)$ we may assume one of $W$ and $Y$ is maximal, while if $F \neq G F(2)$ we may assume that $W$ and $Y$ are both maximal. If $W=Y$ (or if $Y \subset W$ in the case $F$ $=G F(2))$ let $v \in V-(W \cup Y)$ and let $w_{1}, \ldots, w_{n-1}$ be a basis for $W$. Then $B=\{v, v$ $\left.+w_{1}, \ldots, v+w_{n-1}\right\}$ is a basis for $V$ contained in $V-(W \cup Y)$ as desired. 
If $W \neq Y$, then $\operatorname{dim} V+\operatorname{dim}(W \cap Y)=\operatorname{dim} W+\operatorname{dim} Y$. If $F \neq G F(2)$ then $n+\operatorname{dim}(W \cap Y)$ $=2(n-1)$ or $\operatorname{dim}(W \cap Y)=n-2$. Let $w_{1}, \ldots, w_{n-2}$ be a basis for $W \cap Y$. Select $w_{n-1} \in W$, $y \in Y$ such that $\left\{w_{1}, \ldots, w_{n-2}, w_{n-1}\right\}$ is a basis for $W$ and $\left\{w_{1}, \ldots, w_{n-2}, y\right\}$ is a basis for $Y$. Let $v=w_{n-1}+y$, an element of $V-(W \cap Y)$. Let $a \in F^{*}, a \neq 1$, then $B=\left\{v+w_{1}, \ldots, v\right.$ $\left.+w_{n-2}, w_{n-1}+y, a w_{n-1}+y\right\}$ is a basis for $V$ of the desired type.

If $F=G F(2)$ then we may assume $\operatorname{dim} W=n-1, \operatorname{dim} Y=n-2$ and $Y \not \varnothing W$. Let $\left\{w_{1}, \ldots, w_{n-2}\right\}$ be a basis for $W \cap Y,\left\{w_{1}, \ldots, w_{n-2}, w_{n-1}\right\}$ be a basis for $W$, and $\left\{w_{1}, \ldots, w_{n-3}, y\right\}$ be a basis for $Y$. If $v=w_{n-1}+y$, then $B=\left\{v+w_{1}, \ldots, v+w_{n-3}, v+w_{n-2}\right.$, $\left.\dot{w}_{n-2}+y, w_{n-1}+y\right\}$ is a basis of the desired type.

As an application of this lemma, let $V$ be a vector space over the field $F$ and suppose the function $f: V \rightarrow V$ is linear off a proper subspace $W$ of $V$, i.e. $f\left(v_{1}+v_{2}\right)=f\left(v_{1}\right)+f\left(v_{2}\right)$ whenever $v_{1}, v_{2} \in V-W$. If $f \alpha=\alpha f$ for some $\alpha \in$ Aut $V$ let $Y=\bar{\alpha}^{1} W$. From the above lemma there is a basis $B$ for $V$ outside of $W \cup Y$, say $B=\left\{v_{1}, \ldots, v_{n}\right\}$. Let $\beta \in$ Aut $V$ be such that $\beta(x)=f(x)$ for each $x \in V-W$. For $i=1,2, \ldots, n$ we have

$$
\alpha \beta\left(v_{i}\right)=\alpha f\left(v_{i}\right)=f \alpha\left(v_{i}\right)=\beta \alpha\left(v_{i}\right)
$$

since $\alpha\left(v_{i}\right) \notin W \cup Y$. This means $\alpha \beta=\beta \alpha$.

To fix the setting for the next theorem let $V$ be a vector space over a nonprime ficld $F$ with scalar multiplication given by $(a, v)=a v, a \in F, v \in V$. Let $W$ be a nonzero proper subspace of $V$ and let $\sigma$ be an automorphism of $F, \sigma \neq 1$. We define another action $*: F \times V \rightarrow V$ by

$$
a * v=\left\{\begin{array}{c}
a v, v \in W \\
a^{\sigma} v, v \in W .
\end{array}\right.
$$

This gives rise to a subfield $\bar{F}$ of $C(\{1\} ; V)$ where $\bar{F}=\left\{f_{a}: V \rightarrow V \mid f_{a} v=a * v\right\}$. Each $f_{a} \in \bar{F}$ is linear off $W$ and by the above remarks each $\alpha \in$ Aut $_{\mathrm{F}} V$ commutes with the linear maps $\left\{\lambda_{a}: V \rightarrow V \mid \lambda_{a} v=a v, a \in F, v \in V\right\}$. So each $\alpha \in \mathrm{Aut}_{F} V$ is $F$-linear, meaning $C\left(\mathrm{Aut}_{F} V ; V\right)$ contains $\left\{\lambda_{a} \mid a \in F\right\}$. This establishes the following theorem.

Theorem 3.2. Let $F, \bar{F}$, and $V$ be as in the above discussion. Then $\bar{F} \neq C(\mathscr{A} ; V)$ for any $\mathscr{A} \subseteq$ Aut $V$.

\section{REFERENCES}

1. J. C. Beidleman, On Near-rings and Near-ring Modules (Doctoral Dissertation, Penn. State Univ., 1964).

2. G. BETSCH, Primitive near-rings, Math. Z. 130 (1973), 351-361.

3. S. Dancs-Grove, Locally finite near-fields, Abh. Math. Sem. Univ. Hamburg 48 (1979), 89107.

4. I. Herstein, Noncommutative Rings (Carus Monograph 15 MAA, John Wiley and Sons, N.Y., 1965).

5. C. Maxson, M. Pettet and K. SMrth, On semisimple rings that are centralizer near-rings, Pacific J. Math. (to appear).

6. C. MAxson and K. SMrth, The centralizer of a group automorphism, J. Alg. 54 (1978), 27-34. 
7. C. Maxson and K. Sмith, The centralizer of a set of group automorphisms, Comm. in Alg. 8 (1980), 211-230.

8. C. Maxson and K. Smith, Centralizer near-rings that are endomorphism rings, Proc. Amer. Math. Soc. 80 (1980), 189-195.

9. J. Meldrum and A. Oswald, Near-rings of mappings, Proc. Royal Soc. Edinburgh 83A (1978), 213-223.

10. D. Passman, Permutation Groups (W. A. Benjamin, N.Y., 1968).

11. G. PiLz, Near-rings (North-Holland, New York, 1977).

12. D. Ramakotalah, Isomorphisms of near-rings of transformations, J. London Math. Soc. (2) 9 (1974), 272-278.

13. H. WIELANDT, Über Bereiche aus Gruppenabbildungen, Deutsche Mathematik 3 (1938), 9-10.

Department of Mathematics

TEXAS A \& M UNIVERSITY

College Station

TEXAS 77843

U.S.A. 